\title{
MSC ADAMS Based Simulation of a Test-Bed for Cell Injecting Using IPMC
}

\author{
Lina Hao ${ }^{1, a}$, Hongtao Liu, ${ }^{1, b}$, Jianchao Gao ${ }^{1, c}$, Peng Zhang ${ }^{1, d}$ \\ ${ }^{1}$ School of Mechanical Engineering and Automation, Northeastern University, China

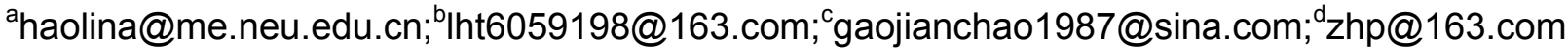

Keywords: Smart material, Virtual prototype, Cell injecting, Dynamic simulation and analysis, PID, Optimization design

\begin{abstract}
The research is aimed at finishing the dynamic simulation of test-bed for cell injecting using IPMC (Ionic Polymer Metal Composites) based on MSC ADAMS. By changing experiment data of the simulation in ADAMS, researchers choose optimization data as the initial parameters of test-bed for cell injecting. A multi-rigid-body system dynamics model of the test-bed for cell injecting is established through analyzing the system dynamic parameters and applying a mechanical dynamic analysis software ADAMS. The optimal PID parameters of microscope's focusing system are confirmed. Thus relevant actuating motor can control microscope's lens rapidly and exactly by these parameters. The realization of ordinal simulation provides accurate recognition of experiment system's initial position and running time. This research finishes dynamic simulation and analysis of a test-bed for cell injecting using ADAMS software. Optimal parameters are chosen for the control system according to simulation and analysis. The theoretical analysis and experimental results can be widely referred by the multi-rigid-body physical prototype manufacture.
\end{abstract}

\section{Introduction}

IPMC (Ionic Polymer Metal Composites) is a kind of novel polymer smart material, which produces large deformation under the low voltage excitation [1]. IPMC is comprised of ion exchange membrane and the noble metal like platinum, gold and silver coated on the both sides of the ion exchange membrane. This material has some features like muscle, such as good-flexibility, low-density and tiny output force. IPMC has a promising application prospect in many industries like aerospace industry, healthy care and human-like robots $[2,3]$. In this paper, a cell is composed of four IPMCs like a paw, thus it is convenient to operate the cell [4].

MSC ADAMS is a kind of software which can be used to make dynamic simulation and analysis for a machine system. It is convenient to virtual prototype of kinematics and dynamic analysis and forecast the performance of the mechanical system, movement range, collision detection, computing the input load of finite element, etc [5].

The design process of dynamic simulation and analysis for cell injecting test-bed is shown in Figure 1. By adjusting the parameters, we can compare many schemes of various parameters, until get the optimal scheme, so as to reduce times of the physical prototype manufacture and test, save costs, shorten the development cycle.

\section{Build Virtual Prototype}

Build 3D model of virtual prototype using Solid Works software which is an extraordinary drawing instrument. In order to simplify the model, under the precondition that there are not errors in the simulation process of virtual prototype, we should reduce the number of machine parts as far as possible, so simplify all the system parts, leave primary moving parts and omit others which are useless to simulation, such as the bolts, gaskets and so on, thus we can add restrictions easily. 


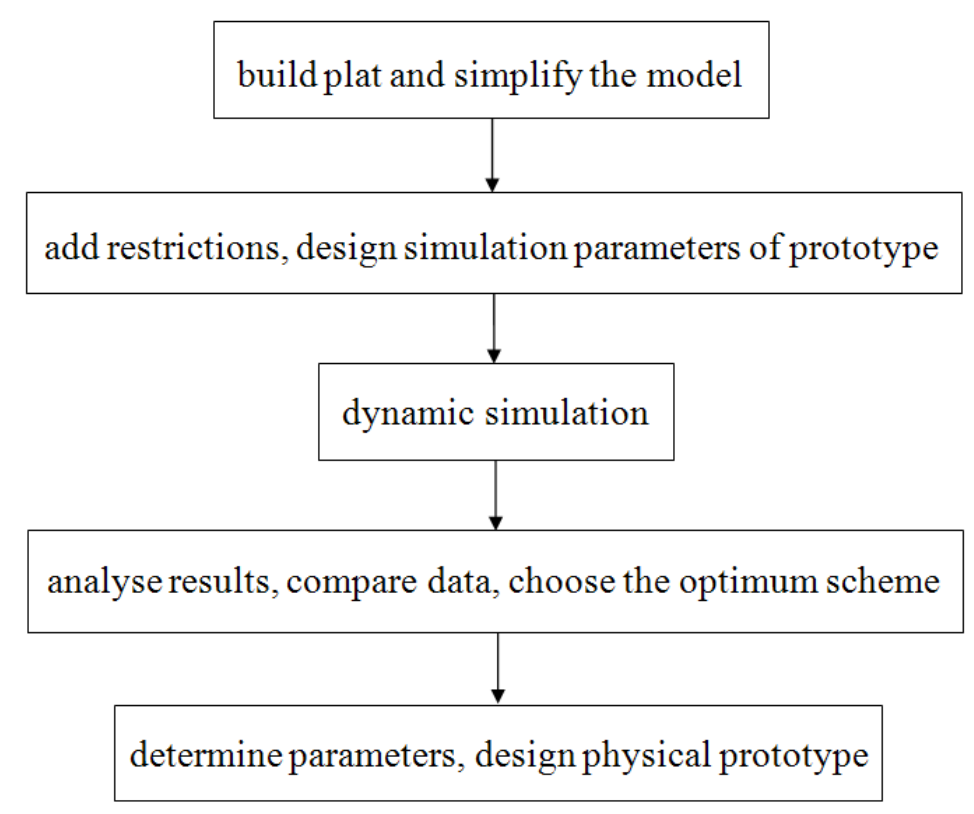

Fig. 1. Design Process of Virtual Prototype

Then guide the model into ADAMS software. In the operating environment of ADAMS, add restriction for every part of the system model. Between microscope's base and ground, the base of three-axis flat and ground, injecting probe and three-axis flat, three-axis flat and three axial DC motor add connection pair restrictions. All scattered parts and their assembly body are connected permanently using merger method. Between each axis add rotation pair restrictions. Between the microscope convergence system and three-axis flat add translation pair restrictions. In the ADAMS software modify the material attribute of every system part, so that the quality, mass center, integral space layout, etc and physical prototype are basically the same [6]. The Virtual Prototype of cell injecting test-bed in ADAMS environment is shown in Figure 2.

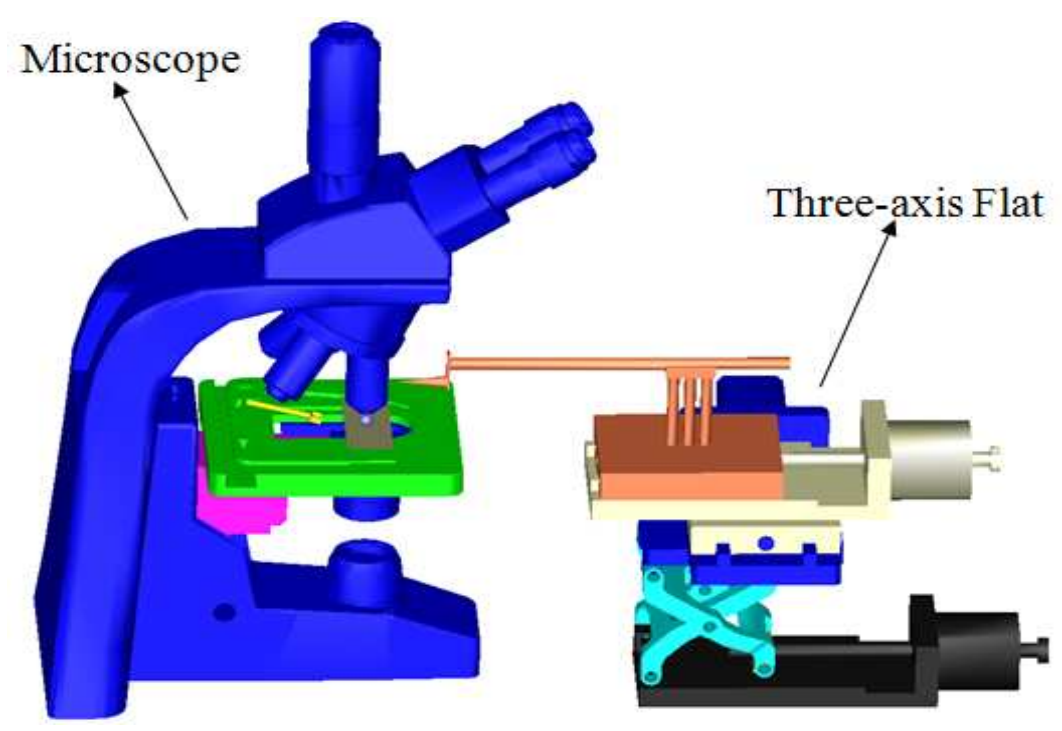

Fig. 2. Model of Virtual Prototype in ADAMS 


\section{Dynamic Simulation of Test-bed}

PID Control Algorithm Based Simulation of Microscope Focus System. The definition of cell and injection probe's images under microscope has a direct impact on the whole experiment system's accuracy, so microscope focusing is the most important in the experiment. Therefore, in the process of simulation, we should make cell and injection probe present the clearest images under microscope in the shortest possible time.

This paper presents a PID algorithm to control microscope focusing. In order to simplify the model, with reference to the actual operation parameters in the microscope, we suppose that when a distance of $6.9 \mathrm{~mm}$ from microscope stage to lens, the cell's image is the clearest. Then this distance is as desired value in the simulation of microscope focus system. Based on the microscope stage's motion, create single component torque in the microscope stage's translation pair and set torque value to zero.

The simulation of microscope focusing system is finished by control toolbox in ADAMS software. Open "Controls Toolkit" toolbox and popup dialog box "Modify Controls Block". At first, click button $f_{i} \rightarrow$, create respectively the microscope stage`s displacement and speed in $Z$ axis as the "Input" and "Derivative Input" of toolbox. Then click button PID and create PID link (shown in Figure3). Finally, click button $\Sigma \mid$ and create compare link. A distance $6.9 \mathrm{~mm}$ which is a preestablished target is as the relative object [7].

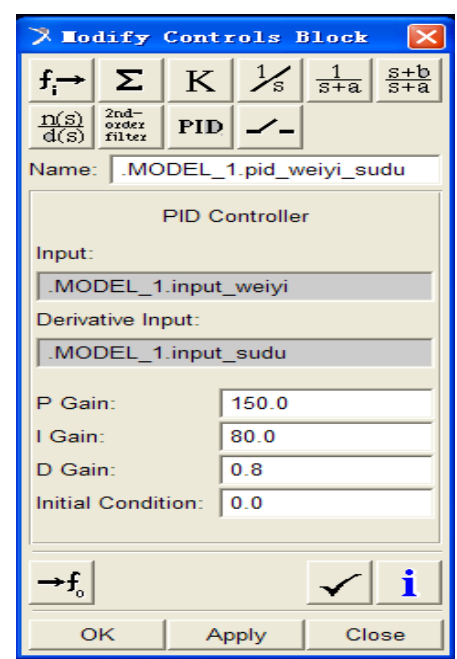

Fig. 3. PID Dialog Box

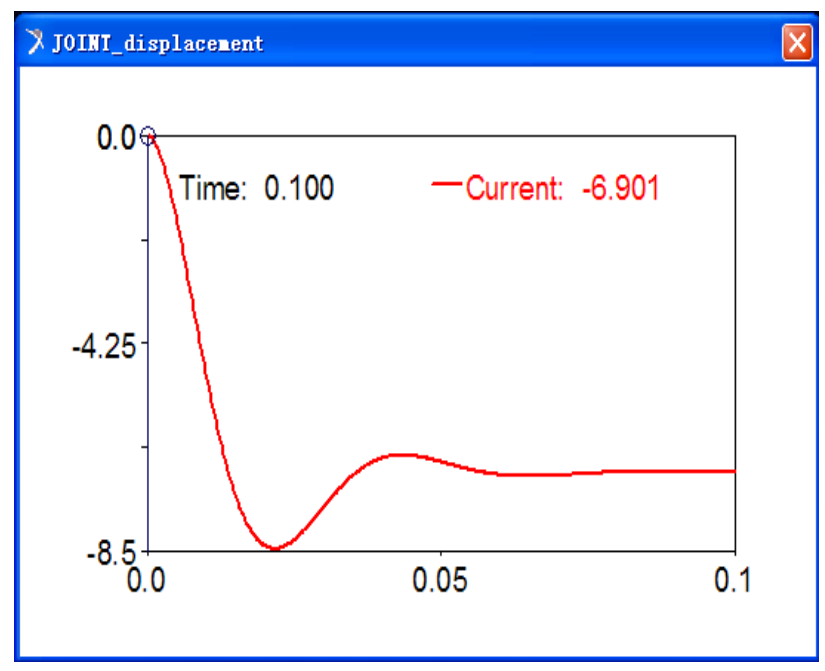

Fig. 4. Displacement Curve of Microscope Stage

Edit single component torque in graph editor area and make them parameterized. Open dialog box "Torque Modify" and choose orderly "Data Element" $\rightarrow$ "Algebraic Variable Value" in the function constructor. In the "Algebraic Variable", choose preestablished compare link as parameter and add "*(-1)" as negative feedback regulating. At last, the function is finished: "VARVAL (.MODEL_1.sum_1.sum_1_input 1$)^{*}(-1)$ ”.

Create displacement measure of microscope stage. After multiple simulations, we choose a group of the most appropriate parameters and determine the PID parameters: P (150), I (80), D (0.8). The displacement curve is shown in Figure4.

From the curve in Figure 4 we can see that microscope's focus system can achieve stable state in $0.1 \mathrm{~s}$ and the error between measured value and desired value is $0.01 \mathrm{~mm}$ which is in the range of allowable. The system overshoot is remained within the microscope's working limits. 
Fasten Cell with IPMC and Simulation of Three-axis Flat. When the cell presents the clearest image under microscope, we use "hand paw" made of IPMCs to clamp cell, preventing cell moving in the process of probe injection. Under the microscope, when the probe touches cell, we can control the voltage to change the deformation of "hand paw" and complete the job of fastening cell [8]. After the cell is fastened, the probe can move forward length by length in the push of three-axis flat driven by three direct current motors in the direction of $\mathrm{X}, \mathrm{Y}$, and $\mathrm{Z}$. The model is shown in Figure5.

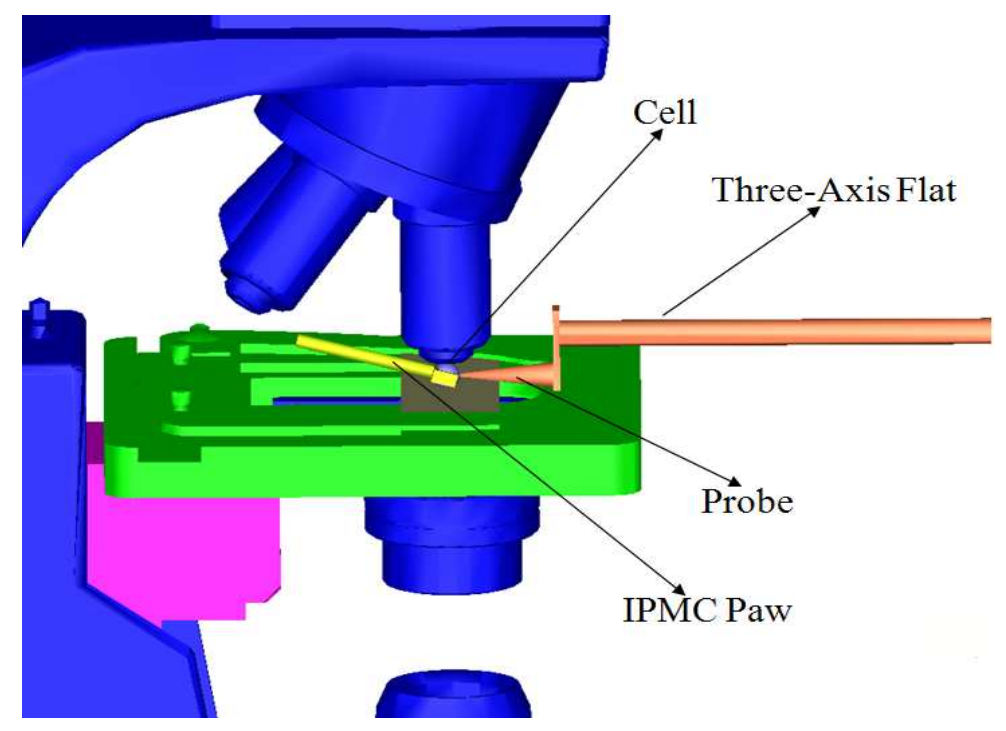

Fig. 5. Model of IPMC Paw and Probe

In order to simplify the model, we omit the control for force and voltage of fastening cell. So we only analyse the injecting process and associated simulation between three axial motions and cell injecting. In the four simulation processes, input the actual numerical value of test-bed, create "Motion" for each translation pair using function "step". The function expressions are shown followed:

1) Process of Fastening Cell: STEP(time,3,-1,3.1,1)-STEP(time, $8,0,8.1,1)$;

2) Process of $X$-axis motion: $\quad-5.75 *(\operatorname{STEP}($ time, $14.99,0,15,1)-\mathrm{STEP}($ time $, 17.69,0,17.70,1)$ );

3) Process of Y-axis motion: $\quad-5.75 *(\operatorname{STEP}($ time, 18.99,0,19,1)-STEP(time,21.69,0,21.70, 1));

4) Process of Z-axis motion: $\quad-6 * \operatorname{STEP}($ time $, 22.69,0,22.70,1)$

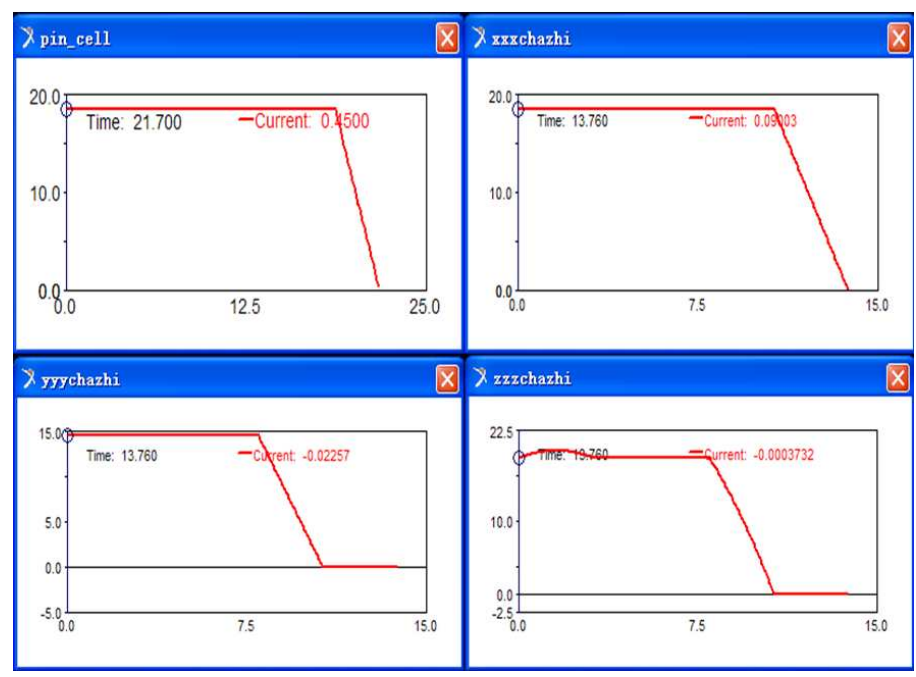

Fig. 6. Associated Simulation Curves 
Create respectively the "Point-Point" measurement for the four processes. The simulation time is $15 \mathrm{~s}$ and the number of steps is 300 . The test curves are shown in Figure6. After simulation, the $\mathrm{D}$-values in four processes turn to zero. That means the injecting work is finished accurately.

\section{Conclusion}

This research finishes dynamic simulation and analysis of a test-bed for cell injecting using ADAMS software and establishes a set of complete design method. The theoretical analysis and experimental results can be widely referred by the multi-rigid-body physical prototype manufacture.

Compared with other simplified design methods, the new design raises mechanical system design accuracy and solves the problem that parameters can't be accurately determined in the design of experimental platform. This method has a great significance for developing system platform rapidly and improving system performance.

\section{Acknowledgements}

This work is supported by The National High Technology Research and Development Program of China (2008AA04Z305), the National Nature Science Foundation of China under Grant 51075066 , the State Key Laboratory of Robotics, (No. RL0200903) and 985 Project of Northeastern University of China.

\section{References}

[1] HAO Li-na, Gao Jian-chao, LIU Hong-tao. A Novel Determination Method of IPMC Young's Modulus based on Cantilever Resonance Theory, C. 2011 International Conference on Mechanical Materials and Manufacturing Engineering. Nanchang, 2011:747-752.

[2] Byungkyo K, Jaewook R, Younkoo J, et al. A Ciliary Based 8-Legged Walking Micro Robot Using Cast IPMC Actuators, C. Proceedings of the 2003 IEEE International Conference on Robotic \& Automation. Taipei, 2003: 14-19.

[3] Thanh T, Vinh K, Youngtai Y, et al. A Novel Polymeric Micropump based on a Multilayered Ionic Polymer-Metal Composite, C. IEEE Industrial Electronics, IECON 2006-32nd Annual Conference on. Paris , 2006: 4888-4893.

[4] Hao Li-na, Xu Su, Liu Bin. A Miniature Fish-like Robot with Infrared Remote Receiver and IPMC Actuator ,J. Journal of Northeastern University (Natural Science), 2009, 30(6): 773-776)

[5] Bai Li-ping. Simulation analysis of robot dynamics based on ADAMS, J. Robot Technology, 2007,7:1001-4551

[6] Rui Zhi-yuan, Wei Xing-chun, Feng Rui-cheng. Virtual prototyping technology based on ADAMS and its application in mechanism design, J. Science Technology and Engineering, 2006, 6 (19) : 3111 - 3114.

[7] NOEL O. Use of ADAMS in Dynamics Simulation of Landing Gear Retraction and Extension, C. 13th European ADAMS User's Conference. France: [s, n], 1998: 1 - 8.

[8] Ran Xian-bin.Design of microforce sensor based on IPMC. School of Mechanical Engineering \& Automation, Northeastern University, Shenyang, 2010. 